\title{
AN UNUSAL CASE OF HIRSUTISM, BALDNESS AND OVARIAN LEIOMYOMA
}

\section{Dr Susie Jacob, Dr Rebecca Lewis, Dr Emma Ward} St James University Hospital, Leeds, UK.

\section{BACKGROUND}

Post-menopausal hyperandrogenism is rare and needs careful evaluation. Unlike the abrupt decline in estrogen post menopause, androgens decline more slowly as the relative increase in gonadotrophins maintain production (Markopoulos et al, 2015).

A differential diagnosis includes: PCOS, non-classical $\mathrm{CAH}$, obesity, ovarian hyperthecosis, endocrinopathies, adrenal tumours, ovarian tumours and iatrogenic causes.

Diagnosis is based on clinical history, physical examination, biochemistry and imaging where appropriate. Treatment depends on cause and will often necessitate some form of surgery or medical therapy.

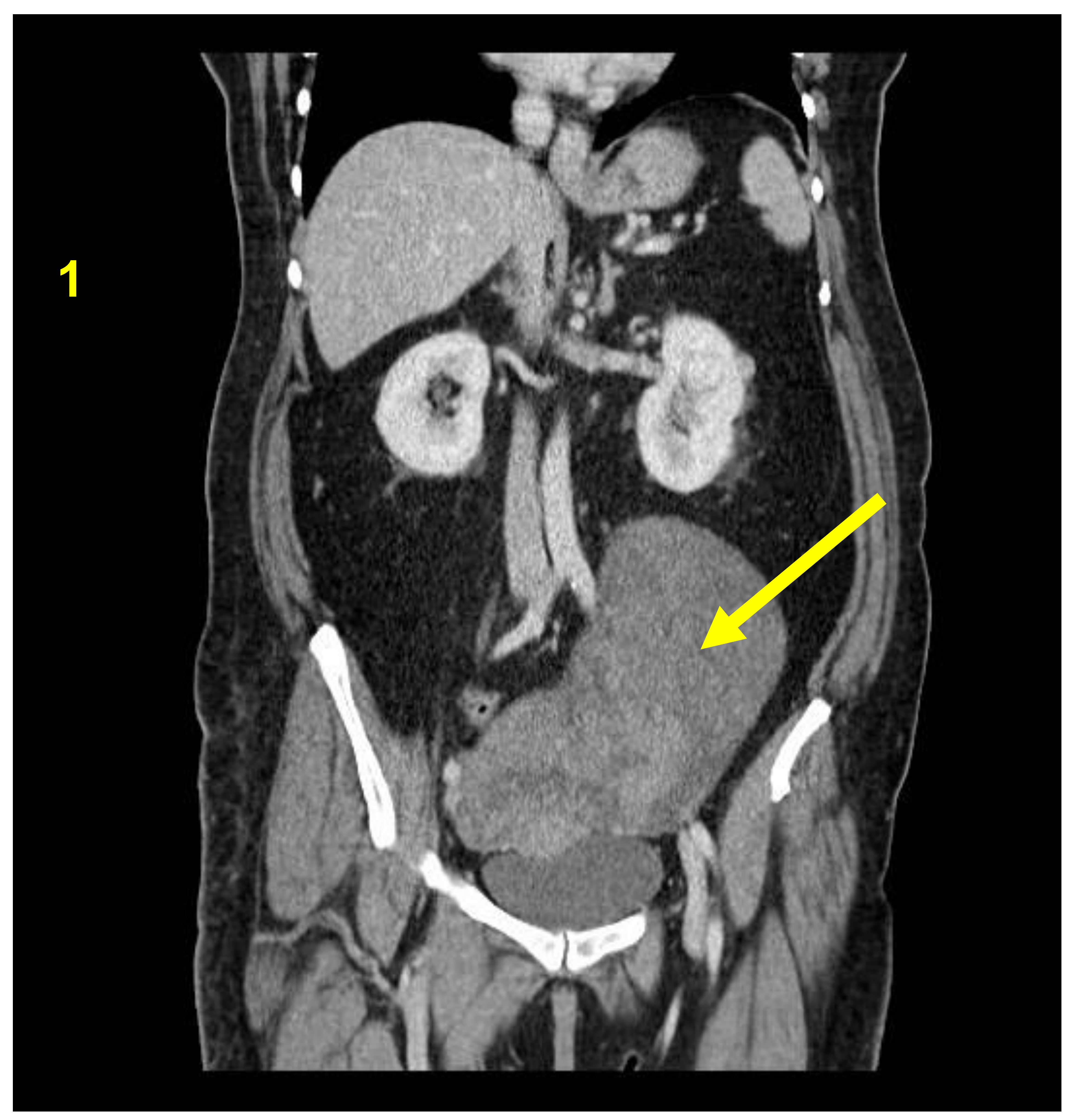

CONCLUSIONS

Ovarian leiomyomas are rare constituting $<1 \%$ of benign ovarian tumours (Faisch et al, 2008). The majority co-exist with uterine leiomyomas. Leiomyomas constitute smooth muscle overgrowth and are not usually related to androgen production. There have only been sporadic reports of virilisation and ovarian leiomyomas in the literature. Androgen production in conjunction with ovarian leiomyoma, is considered secondary to thecal cell irritation by tumour growth. This case serves to remind that androgenic symptoms in the postmenopausal woman may be related to ovarian tumours and should be considered in the differential diagnosis. Surgical treatment is highly successful, returning most women to their norm. Incidentally, the renal masses in this case may reflect a wider case of leiomyomatous disease.
Leeds Centre for

Diabetes and Endocrinology
A 60-year-old woman presented to the endocrine clinic with significant hirsutism and male-pattern baldness, progressive since the menopause 5 years earlier. She was otherwise fit and well. Testing revealed an elevated serum testosterone of $14.2 \mathrm{nmol} / \mathrm{L}$. A CT scan revealed a large malignant $19 \mathrm{~cm}$ mass arising from the left adnexa, a large fibroid uterus and 2 small masses in the left kidney. Other abdominal organs were normal with no visible ascites.

With the presumption of malignancy, she underwent a staging laparotomy under the gynaecology oncology team, which included a total abdominal hysterectomy and bilateral salpingo-oophorectomy.
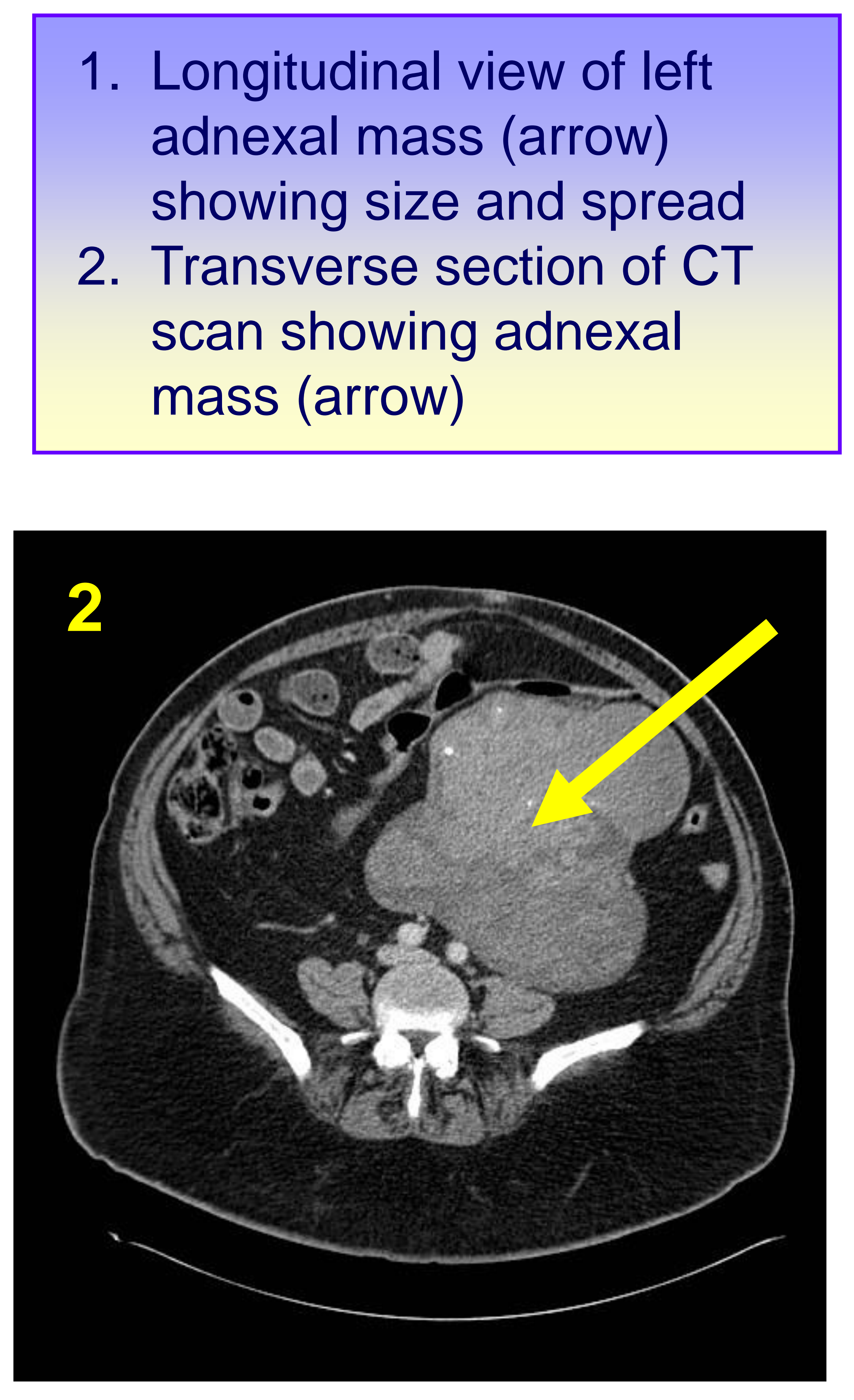

References

- Faisch, $N$ et al. Leiomyomas beyond the uterus:unusual locations, rare manifestations. Radiographics (2008) 28:1931 1948

-Markopoulos, $M$ et al.

Hyperandrogenism after

menopause. European Journal of Endocrinology (2015) 172, R79 R91

SfE BES, Glasgow 2018 